Topiques, études satoriennes

Topoï Studies, Journal of the SATOR

\title{
De la Delie de Scève en Diotima rediuiua, ou l'image d'un mentor sans le son
}

\section{Xavier Bonnier}

Volume 4, 2018

Maître-disciple : une relation topique

URI : https://id.erudit.org/iderudit/1074719ar

DOI : https://doi.org/10.7202/1074719ar

Aller au sommaire du numéro

Éditeur(s)

SATOR, Société d'Analyse de la Topique Romanesque d'Ancien Régime

ISSN

2369-4831 (numérique)

Découvrir la revue

Citer cet article

Bonnier, X. (2018). De la Delie de Scève en Diotima rediuiua, ou l'image d'un mentor sans le son. Topiques, études satoriennes / Topoï Studies, Journal of the SATOR, 4, 1-17. https://doi.org/10.7202/1074719ar
Résumé de l'article

Mentor exceptionnel car femme, étrangère, et instruisant Socrate, Diotime constitue à la Renaissance une référence majeure pour réfléchir sur l'amour en rejouant le Banquet. Mais tout se complique lorsque Scève, après Castiglione et Speroni, campe Diotime en métaphore de l'Aimée dans Delie (1544) : la concurrence d'autres instances féminines, le caractère final de l'assimilation, l'ambiguité structurelle du dizain, et le caractère silencieux d'un discours prétendument rédempteur, laissent apparaître une dimension ironique, car Diotime enseigne aussi bien à haïr qu'à aimer, et la spécificité retorse d'un discours qui préfère l'inscription à l'expression. 


\section{De la Delie de Scève en Diotima rediuiua, ou l'image d'un mentor sans le son}

À un double titre - l'un, certes, plus ludique que l'autre -, le propos qui va suivre transgresse le cadre et les réquisits du colloque Mentors and Mentees, dont l'argumentaire dit en effet d'une part que « le mentorat n'est pas genré », d'autre part que « les femmes peuvent jouer un rôle de guide moral et social» : en jouant sur la polysémie du substantif «genre » et en le prenant au sens littéraire, il est possible d'objecter au premier postulat que le mentorat ne s'inscrit d'ordinaire, ou très majoritairement, que dans les œuvres narratives en prose, la poésie, et plus encore la poésie amoureuse en recueil, n'étant a priori vraiment pas la mieux placée pour proposer une formation dans le temps, un cheminement initiatique, une transmutation de la personnalité inspirée par une référence respectée. Or, il sera question d'un recueil poétique sans réel fil conducteur, sans histoire linéaire, mais où figure un mentor, ce qui va confirmer paradoxalement le postulat de l'absence d'assignation générique. Sur le second point, où, cette fois, le mot « genre » garde son acception biologique et sociale, sera examinée une figure féminine extrêmement célèbre, et dont il est hors de doute que lui est reconnu ce rôle de guide, mais dont tout concourt à brouiller le message et le sens de son apparition dans l'œuvre qui l'évoque explicitement, en lien avec le personnage central. C'est en effet de Maurice Scève, le grand poète lyonnais, dont il faut rappeler qu'il fait en quelque sorte la transition entre Marot et la Pléiade dans les années 1540, qu'il va s'agir. Son chefd'œuvre, Delie, object de plus haulte vertu, paru à Lyon en 1544, est le premier canzoniere en langue française, constitué de 449 dizains décasyllabiques et rythmé par cinquante « emblèmes » qui se succèdent de neuf dizains en neuf dizains depuis l'intervalle entre le cinquième et le sixième jusqu'au seuil de l'antépénultième ${ }^{1}$. Si la bibliographie sur ce recueil dense et exigeant est trop massive pour qu'on se risque à en résumer les acquis s'agissant de ce qu'il faut bien appeler le projet du poète, son intention ou son message, il peut suffire de rappeler que l'Amant-poète, qui ne se désigne jamais en tant que Maurice Scève, ni sous aucune autre identité civile plausible, gratifie son Aimée d'un certain nombre d'avatars ou de

\footnotetext{
${ }^{1}$ Le terme d' « emblème » est ici employé par commodité, car le véritable livre d'emblèmes, sur le modèle du grand succès d'Alciat (Emblematum liber, éd. princeps chez Steyner à Augsburg, 1531, $1^{\text {re }}$ éd. fr. chez Christian Wechel, Paris, 1534), et que reprendront Corrozet, La Perrière, etc., est un genre didactique et moral à part entière, en pleine expansion au XVI siècle, et dont Scève détourne malicieusement la formule de base en reprenant dans un contexte de recueil amoureux la triade [vignette gravée - devise ou motto - poème d'accompagnement].
} 
personce, et cela tout au long du recueil, de temps à autre, sans vraie régularité. Que «Delie » ait constitué ou non le masque littéraire, le senhal, de Pernette du Guillet ou d'une pluralité de personnages féminins avérés dans le milieu cultivé lyonnais de l'époque, est une question très secondaire, et rien de probant n'a été avancé à ce sujet ${ }^{2}$.

Or, dix dizains seulement avant la fin du recueil, et au terme d'une série d'identifications qui seront rappelées car elles jouent leur rôle, Scève écrit, au D 439 :

\footnotetext{
Bien que raison soit nourrice de l'ame,

Alimenté est le sens du doulx songe

De vain plaisir, qui en tous lieux m'entame,

Me penetrant, comme l'eau en l'esponge.

Dedans lequel il m'abysme, \& me plonge

Me suffocquant toute vigueur intime.

Dont pour excuse, \& cause legitime

Je ne me doibs grandement esbahir,

Si ma tressaincte, \& sage Dyotime

Tousjours m'enseigne a aymer, \& hair ${ }^{3}$.
}

La mention explicite de Diotime n'est pas un mince point d'appui pour ceux qui, comme P. Martin et d'autres, soutiennent la thèse du néoplatonisme de Scève, un néoplatonisme certes tempéré et qu'il faut bien circonscrire ${ }^{4}$, mais un néoplatonisme tout de même assez militant et donnant tout son sens au recueil. À si peu de distance de la fin de celui-ci, l'affaire paraît d'ailleurs entendue: Délie est pour l'Amant une nouvelle Diotime qui lui enseigne le véritable amour, comme elle l'a enseigné à Socrate dans Le Banquet, et le lecteur peut supposer que la passion subie par l'Amant depuis le traumatisme de l'innamoramento débouche sur une élévation vers le Beau en soi telle que l'explique à Socrate la femme de Mantinée. L'inspiration largement platonicienne du propos de l'Amant-poète a déjà été abondamment documentée, et entre maints détails on peut rappeler la mention de « L'hermaphrodite, efficace amoureuse ${ }^{5} », \mathrm{du}$ « Ciel, non Peripatetique ${ }^{6} »$, ou encore de la beauté de Délie qui est adorée comme «esmerveillable Idée ${ }^{7}$ », soit autant de vocables symptomatiques immédiatement reconnaissables, et employés du reste dans un cotexte qui confirme une obédience au moins partielle. La traversée des épreuves de la passion

\footnotetext{
${ }^{2}$ Précisons au passage qu'en vertu d'une convention récente, Delie est en italiques dès qu'il s'agit de tout ou partie du titre du recueil, mais que pour évoquer le personnage féminin lui-même, le choix est ouvert entre le classique « Délie » et le plus orthodoxe « Delie ».

${ }^{3}$ Maurice Scève, Délie object de plus haulte vertu, texte établi et annoté par Eugène Parturier, introduction par Cécile Alduy, p. 297-298. Toutes les citations sont tirées de cette édition.

${ }^{4}$ Comme le fait par exemple Michael J. Giordano dans The Art of Meditation and the French Renaissance Love Lyric. The Poetics of Introspection in Maurice Scève's Délie, Object de plus haulte vertu (1544).

${ }^{5}$ D 435 , v. 6.

${ }^{6}$ Donc non aristotélicien, donc implicitement platonicien (D 444, v. 1).

${ }^{7}$ D 275, v 2. Voir aussi le D 2 : «Le Naturant par ses haultes Idées ». Le terme (qui n'est employé cependant qu'à deux reprises) est toujours en majuscule.
}

https://journals.uvic.ca/index.php/sator/index 
amoureuse a ainsi pu être interprétée, non sans mal il est vrai pour la fin du recueil, même si elle promet l'immortalité, comme une lente et pénible ascension de l'obscurité vers la lumière du véritable amour, et même si les travaux les plus récents ont mis à mal cette thèse essentiellement dix-neuviémiste, elle offre une belle résistance et trouve encore des partisans. Enfin, il est difficile de nier la quasi-divinisation de Délie, son grandissement par l'Amantpoète, sa mythification, qui peut bien être le préalable de l'effort d'élévation spirituelle vers le monde des Idées.

Et pourtant, tout ne va pas de soi dans ce dizain 439, et sur plusieurs plans, complémentaires et non contradictoires, il est nécessaire de le réexaminer, en commençant par le simple niveau grammatical : comme souvent dans les dizains de Scève, une dynamique prédicative oppose un sizain et un quatrain, alors que le schéma des rimes oppose plutôt deux quintils, selon un mélange qui a été déjà largement étudié ${ }^{8}$, et cette compensation d'une indépendance sur un plan (phonétique) par une dépendance sur l'autre (syntaxique), ce mixte de liaison et de déliaison, fait partie des charmes du recueil ${ }^{9}$. Ici, comme c'est fréquemment le cas, c'est un adverbe de discours, le « Dont» du v. 7, qui assure la transition. Ce relatif englobant signifie «Et c'est pourquoi », « et c'est ce qui explique que », «et, par conséquent ${ }^{10}$. Mais cette clarté sémantique locale n'empêche pas que deux interprétations du rapport entre sizain initial et quatrain final restent possibles.

D'un côté en effet, le sizain est apparemment ce qui explique et justifie le quatrain. Une paraphrase de ce que dit ici l'Amant pourrait restituer l'ensemble ainsi : «Bien que la raison (donc la capacité à être lucide et maître de soi) nourrisse l'âme, ma sensibilité, elle, se nourrit d'une rêverie très agréable, de fantasmes sur un plaisir dont je sais bien qu'en fait il est vain, mais qui partout m'obsède, m'envahit, et me plonge dans ce vain plaisir, en me privant de mes moindres forces. C'est pourquoi, ce phénomène constituant en soi une excuse et une cause légitime, je ne dois pas m'étonner si la très sainte et sage Délie, telle une nouvelle Diotime, m'enseigne à aimer et hä̈r (donc veuille me ramener sur le droit chemin, à la raison) ». Dans ce premier cas, la «formation » que Délie / Diotime fait subir à l'Amantpoète est envisagée comme solution - peut-être déjà engagée au demeurant, mais peut-être aussi à venir, car le «tousjours m'enseigne » est d'aspect sécant -, de cet égarement

\footnotetext{
${ }^{8}$ Voir notamment Edwin M. Duval, «From the Chanson parisienne to Scève’s French canzoniere : Lyric Form and Logical Structure of the Dizain », dans A Scève Celebration: Délie 1544-1994, p. 71-85, et Cécile Alduy, "Les "erreurs" de la versification dans Délie de Maurice Scève ", Nouvelle Revue du Seizième siècle, n 16/2, 1998, p. 249-266.

${ }^{9}$ Ici en l'occurrence, la structure phrastique est duelle : Sizain $(=\mathrm{ababbc}) \rightarrow($ Quatrain $=\mathrm{cdcd})$. La rime c assure donc une passerelle sensorielle par dessus la coupure phrastique.

${ }^{10}$ Huguet dit «À cause de quoi, par suite de quoi », et donne de nombreux exemples, dans son Dictionnaire de la langue française du XVI $I^{e}$ siècle, Classiques Garnier Numériques, ad loc.
} 
libidineux; autrement dit, l'Amant-poète expliquerait ici que, si Délie joue un rôle d'initiatrice aux véritables valeurs de l'amour, c'est parce que lui, l'Amant, est faible et se laisse égarer par sa libido. Mais dans cette hypothèse, pourquoi ajouter une initiation à la haine, qui assurément perturbe la sensibilité au lieu de l'apaiser ? Remettons provisoirement les réponses possibles à cette question, car il faut examiner en priorité l'autre possibilité logique.

D'un autre côté, la causalité pourrait être en sens inverse, le quatrain final expliquant rétrospectivement le sizain : Délie se comportant vis-à-vis de l'Amant-poète comme une nouvelle Diotime, sainte, sage, et consciencieuse initiatrice au véritable amour, il n'est pas étonnant qu'il soit dévoré par la concupiscence. La paraphrase serait cette fois : « Bien que la raison (donc la capacité à être lucide et maître de soi) nourrisse l'âme, ma sensibilité, elle, se nourrit d'une rêverie très agréable, de fantasmes sur un plaisir dont je sais bien qu'en fait il est vain, une rêverie qui partout m'obsède, m'envahit, et me plonge dans ce vain plaisir, en me privant de mes moindres forces. De ce phénomène, il n'est pas étonnant que je trouve une excuse et une cause légitime dans le fait que la très sainte et sage Délie, telle une nouvelle Diotime, m'enseigne à aimer et haïr (donc veuille me ramener sur le droit chemin, à la raison, alors que j'ai foncièrement envie d'autre chose) ». Dans ce cas-là, la « haine » du dernier vers est problématique, mais moins que dans le premier.

Ce genre d'incertitude n'est pas rare dans le recueil: nombreuses sont les occasions d'hésitation entre une restitution syntaxique et une autre, surtout lorsqu'il y a antéposition possible d'un complément d'objet, ou flottement référentiel d'un pronom. Le cas est typique ici, puisque malgré l'apparente solidité de la construction -et de nombreux travaux ont relevé la subtilité grammaticale de Scève, qui pastiche parfois les raisonnements juridiques en deux ou trois temps-, le lecteur est bien empêché de savoir si la formule «pour excuse, \& cause legitime » est anaphorique (c'est la première solution, qui sous-entend à peine un verbe «être» qui l'applique à tout ce qui précède), ou cataphorique (seconde solution, qui simplement annonce et résume par avance le quatrain).

Il est évidemment toujours frustrant de ne pouvoir trancher avec certitude au regard du cotexte et du contexte : les partisans de l'option néoplatonicienne privilégieraient la première lecture, Délie dispensant la bonne doctrine du véritable amour en réponse thérapeutique au désordre émotionnel de l'Amant. Ceux qui, au contraire, préfèrent voir dans le recueil une entreprise hétérodoxe et expérimentale, éminemment éclectique, pragmatique et subversive, penchent pour la seconde solution. Ce qui paraît devoir emporter la décision en faveur de cette seconde option, c'est le choix des vocables « excuse » et « cause legitime » : ils sont tout https://journals.uvic.ca/index.php/sator/index 
de même moins propres à fonder l'entreprise curative de Délie (pourquoi aurait-elle besoin d'une « excuse » pour lui enseigner sa sagesse alors qu'il est le jouet des passions ?), qu'à qualifier son effet secondaire indésirable et délétère, sa douloureuse contrepartie. La question peut rester néanmoins en suspens, puisque l'essentiel n'est pas exactement dans ce rapport de cause à effet, mais dans le type de magistère qu'exerce Délie sur l'Amant-poète : après tout, que ses leçons soient la réponse à la torture libidinale de l'Amant, donc une cure bienfaisante, ou son déclencheur contre-productif, il y a bien une association à Diotime qu'il convient d'éclaircir, en commençant par la nature de ces « leçons ».

Et donc par le verbe « enseigner », crucial en cette affaire, et qui est présent à deux autres reprises dans le recueil : au D 40, où le sens et le cotexte sont tout à fait proches :

Quiconques fut ce Dieu, qui m'enseigna

Celle raison, qui d'elle me revoque,

D'un trop grand bien, certes, il me daingna :

Pource qu'a mieulx ma voulenté provoque ${ }^{11}$.

À condition d'entendre « revoquer» au sens de «détourner, faire revenir $»^{12}$, le lecteur comprend que le dieu Amour, fort proche en cela de la Diotime à venir, a enseigné à l'Amantpoète un usage de la raison qui l'éloigne de Délie pour son plus grand bien, et oriente sa volonté vers de plus nobles objectifs que celui que l'on devine, purement sensuel. La suite du dizain précise d'ailleurs que c'est la liberté de Délie qui est préservée par cette instruction de la raison ${ }^{13}$.

La seconde occurrence est plus significative encore, et fait apparaitre une source livresque très éclairante ; à peine trois dizains auparavant, le D 436 débute ainsi :

Incessamment travaillant en moy celle,

Qui a aymer enseigne, \& reverer,

Et la suite est très positive. La relative périphrastique désignant Délie est directement inspirée de Pétrarque, qui qualifie Laure de "Quella ch'amare et sofferir ne 'nsegna », autrement dit

\footnotetext{
${ }^{11}$ Le soulignement d'« enseigna » par des italiques est de mon fait, comme tous les soulignements ultérieurs à l'intérieur du texte scévien.

${ }^{12} \mathrm{Mc}$ Farlane le comprend à tort au sens de « rappeler, faire se souvenir » (The « Délie » of Maurice Scève, p. 383), ce qui ne cadre pas aussi bien avec la suite que le sens de « détourner». Cela dit, la mise en vedette du travail de la mémoire est l'un des aspects majeurs du discours de l'Amant dans le recueil, l'un des rares résultats probants de l'expérience amoureuse (voir mes «Éléments de psychérologie scévienne », publiés en ligne sur le site Fabula :

http://www.fabula.org/colloques/document1805.php). G. Defaux est probablement dans l'erreur en parlant de «l'échec» de l'Amant (Delie object de plus haulte vertu, t. II, p. 70).

${ }^{13}$ « Car loy d'Amour est de l'un captiver, / L'aultre donner d'heureuse liberté » (v. 9-10).
} 
« celle qui à aimer et souffrir nous apprend ${ }^{14}$ ». Il y a une vertu initiatique de Laure, avant la conversion de son Amant. La variante scévienne tranche donc avec violence, comme l'avait remarqué il y a quarante ans Doranne Fenoaltea : « in D436, wholly admiring, the lover says that his beloved « a aymer enseigne, \& reverer »; in D439, faced with the darker side of his passion as well, he corrects the earlier expression to «Tousjours m'enseigne a aymer, \& hair ». Whether the object of his love and hate is his love or Délie is not entirely clear ${ }^{15} » . \mathrm{Il}$ est temps en effet de revenir sur cette notion de haine, qui non seulement, comme ici, opère une déviation capitale par rapport à un texte-source majeur pour Delie, mais apparaît de manière délibérément spectaculaire et provocatrice sous la forme d'une chute, ou d'un concetto, en bathos ${ }^{16}$. Car le lecteur / auditeur s'attend à tout comme dernier mot, dans cette présentation dithyrambique d'une nouvelle Diotime, sauf à ce rugueux verbe « haïr », dissimulé après l'ultime virgule, comme tapi en attente d'un surgissement qui compromet rétrospectivement tout l'édifice patiemment édifié jusque-là. Depuis quand la spécialiste légendaire du véritable amour donne-t-elle des cours de haine ? Et ceux-ci sont-ils même concevables? Car curieusement, le public d'hier et d'aujourd'hui conçoit aisément que l'amour puisse exiger un réel apprentissage, pour dépasser les apparences illusoires ou les appétits basiques, mais justement parce qu'il s'agit d'un trajet de type éducatif, alors que la haine est sans doute ce qui demande le moins d'apprentissage, car elle procède d'un instinct qu'il faut justement apprendre à juguler. Le contraste avec Pétrarque, en cette occasion comme en d'autres, est saisissant et ne pouvait passer inaperçu.

Mais une autre source avérée permet peut-être de surmonter la contradiction, le Dialogo d'amore de Sperone Speroni. Ce dialogue à trois voix, paru à Venise en $1542^{17}$, et que Scève a lu de très près - comme Du Bellay lira de très près le Dialogo delle lingue pour écrire la Deffence -, part du problème de la jalousie amoureuse pour mettre progressivement en évidence le rôle capital de la raison et de la mémoire dans le véritable amour, mais aussi celui du souvenir assumé des satisfactions charnelles : le vrai meneur de jeu, le rationaliste et subtil Gratia, réfute doucement les points de vue inexpérimentés de Tasso et Tullia, qui vont devoir vivre loin l'un de l'autre pendant longtemps et se demandent si leur inquiétude jalouse est bien de l'amour; et comme le rappelle P. Martin, dans son édition du texte et de la traduction

\footnotetext{
${ }^{14}$ Canzoniere / Le Chansonnier, éd. bilingue de P. Blanc, sonnet 140, v. 5, p. 276-277.

15 "The Final Dizains of Scève's "Délie" and the "Dialogo d'Amore" of Sperone Speroni », Studi Francesi n 59, 2, maiaoût 1976, p. 215.

${ }^{16}$ Le « vrai » bathos suppose une gradation préalable, particulièrement énumérative (voir B. Dupriez, Gradus - Les procédés littéraires (Dictionnaire), p. 92). Rien de tel ici, mais l'effet de renversement joue à plein au terme d'un quatrain baigné d'irénique adoration.

${ }^{17}$ Grâce aux soins de son ami le Cardinal humaniste, ambassadeur et grand scientifique Daniele Barbaro.
}

https://journals.uvic.ca/index.php/sator/index 
française qu'en a donnée Claude Gruget en 1551, c'est en réalité le discours platonicien orthodoxe d'un quatrième personnage absent, Molza, repris trop ingénument par Tullia, qui est mis à distance et miné de l'intérieur par Gratia à coup de questions faussement naïves ${ }^{18}$. Non seulement il est patent que Scève reprend des passages littéraux de Speroni, mais on sait depuis les éditions historiques (à commencer par celle de Parturier en 1916) que les dizains 424 à 446 de Délie sont étroitement inspirés par l'œuvre du Padouan, à la limite du démarquage, au point que l'on parle de la «séquence Speroni » pour la fin (ou la «presque fin ») du recueil. Or, il est bien question de haine dans ce dialogue, en des termes et dans un contexte qu'il faut très précisément rappeler : s'efforçant de montrer que l'amour vrai se passe de jalousie, qui selon lui conduit à la haine ${ }^{19}$, Gratia demande à Tullia de bien vouloir expliquer comment l'amour peut susciter la haine de la personne aimée, alors que, et il est très important d'en tenir compte, Tullia est une courtisane au sens le plus raffiné du terme - mais une courtisane parfaitement consciente de son indignité par rapport à Tasso et au reste de la société recommandable, inspirant l'amour sans être à égalité -, et qu'elle soutient que l'amour est d'origine fatale et divine, sans offrir de prise à la volonté. Pour Gratia, Tullia est la digne successeuse de Sappho et de Diotime, et doit accepter de parler en quelque sorte à leur suite et en leur nom :

\begin{abstract}
Degnate adunque d'essere la terza in numero, fra cotanto valore $; \&$ di tai nostri ragionamenti pregate Amore che en componga una novelletta, ove il vostro nome si scriva. Non altramente, che ne dialoghi di Platone, si faccia quello di Diotima, laqual cosa, acciò si faccia con vostra gloria, insegnateci in che maniera l'amante, amando la cosa amata, muova lei ad amare, \& come esser possa, che alcuna volta la cosa amata, amando, odii et voglia male all'amante...

Soit, sous la plume de Gruget: «Veuillez donc, [TULLIE], ne desdaigner d'estre la troisiesme en nombre $[\ldots]$ Et priez amour, que de noz propoz, soit composée une nouvelle, où vostre nom soit escrit : Tout ainsi que Diotime aux dialogues de Platon : Et à fin que cela se face en vostre honneur, enseignez nous en quelle sorte, l'Amant en aymant la chose aymée, l'esmeut à aymer, \& comment il se peult faire, que quelquefois la chose aymée, en aymant porte haine \& malveillance à l'amant. $»^{20}$.
\end{abstract}

Dans un développement aussi long que confus, et plus analogique que syllogistique, Tullia explique alors, par une prolepse qui attribue cette opinion à Gratia et à tous les rationalistes, que l'Aimée, même si elle se sent flattée par les sentiments de l'Amant, se trouve rarement comblée à la mesure de ce qu'elle croit mériter, et, lorsqu'elle fait le compte des qualités de ce même Amant («e notando con diligenzia d'una in una le condizion dell'amante »), peut s'estimer lésée et choisir donc de ressentir de la haine à son égard (« elegge alla fine d'averlo

\footnotetext{
${ }^{18}$ Voir Sperone Speroni, Dialogo d'amore, traduit par Claude Gruget, Dialogue traittant d'amour \& jalousie, Poitiers, 1998.

${ }^{19}$ « Aussi encor qu'un jaloux soit amoureux, toutesfois Jalousie est le chemin, qui plutost conduit à la / haine, qu'à l'amour » (éd. cit., p. 77-78; éd. orig. $\mathrm{f}^{\circ} \mathrm{A} 4 \mathrm{r}^{\circ}$ ).

${ }^{20}$ Éd. cit., p. 132-133.
}

https://journals.uvic.ca/index.php/sator/index 
in odio », p. 136, $\mathrm{f}^{\circ} \mathrm{D} 7 \mathrm{r}^{\circ}$ ), exactement comme les Romains de l'Antiquité faits prisonniers se suicidaient par haine de la servitude ; au contraire, à son avis à elle, l'amour est un arrêt fatal qui ne se laisse pas enfermer dans les manœuvres humaines et le libre-arbitre : à son corps défendant, celui qui aime tend à l'Aimée un miroir concave qui brûle et éblouit au lieu d'éclairer (car elle tient que chacun se connaît dans le regard de l'autre). Bien entendu, ce qu'elle exprime ici est à appliquer, dans la scénographie énonciative, en inversant les sexes par rapport au schéma habituel du soupirant et d'une Dame qui le jugerait indigne d'elle, puisque la personne aimée qui s'estime flouée ne peut être en l'occurrence que Tasso, le grand poète qui la fréquente avec passion, alors qu'elle-même, Tullia, simple courtisane même si elle est de très haut vol, ne peut proposer un tissu de «condicions » («le condizion dell'amante », ibid.) qui fasse jeu égal et mérite sa constance. Par la suite, le sujet des discours dérive, et ne revient pas sur ce point. Bref, si la notion de haine est présente dans le Dialogo d'amore, c'est à titre de risque encouru par des amants trop disparates, un risque tout théorique, parti d'une question a priori réellement ouverte de Gratia, et qui a reçu une réponse que Tullia elle-même n'assume pas. En tout cas, il ne s'agit absolument pas d'un traitement paritaire de l'amour et de la haine, ni d'une assignation de celle-ci à l'Amant, ce qu'avait bien remarqué Doranne Fenoaltea : "The Diotima reference is similarly transformed: Gratia asks why the beloved loves and hates, but Scève speaks instead of the lover's paradoxical attitude. ${ }^{21} \gg$.

Et d'esquisser une explication intéressante :

The sources are thus used with great freedom, in part perhaps because the focus of the dizain-desireis generally absent from the considerations of the dialogue, where its protagonists discuss love beyond an assumed physical satisfaction of desire. ${ }^{22}$.

Ce n'est vrai que globalement, car par exemple dans la suite du dialogue s'élève un débat entre Tasso et Gratia sur la question de savoir qui éprouve le plus fortement l'amour, de l'homme ou de la femme, et la nature même du désir est franchement débattue, en termes de manque et de complémentaritée ${ }^{23}$. Mais là n'est pas l'important : il faut savoir dans quelle mesure Délie est une Diotime, et quelle est la nature de cette haine qu'elle est censée enseigner à l'Amant-poète.

Il faut donc revenir à la source majeure, Platon lui-même et son Banquet, qui met en scène, après toute une série d'interventions et d'opinions exprimées sur la nature de l'amour, le

\footnotetext{
21 «The Final Dizains of Scève’s Délie », art. cit., p. 216.

22 Ibid.

${ }^{23}$ Ibid.., p. 141-145.
} 
personnage de cette étrangère qui a tout appris à Socrate en cette matière. Il le faut d'autant plus que tout le monde n'a pas remarqué cet ajout bizarre de la haine, contrairement à Alison B. Lovell, qui dit :

Dans le Banquet, Diotime n'enseignait pas la haine à Socrate, mais l'amour, donc on peut penser que cette idée ne provient pas directement du Banquet. Cependant Speroni fait allusion à l'amour et à la haine [...] Délie serait la Dyotime de Scève, c'est une de ses métamorphoses (Délie est tour à tour Hecate, Dictynne, Diane / Artémis, et al.) : elle le guide et l'instruit en amour, mais ce ne sont pas les mêmes leçons que celles données par Diotime à Socrate dans le Banquet. Délie / Dyotime est une présence constante auprès de Scève, elle lui enseigne à aimer, bien qu'elle ne l'aime pas forcément avec la même intensité. Le vers 10 du dizain 439 rappelle le Carmen 85 «Odi et amo» de Catulle et l'oxymore amour-haine sur lequel il se base, où le bien-aimé est ami et ennemi à la fois, et où il y a une tension entre la guerre et le désir d'union. ${ }^{24}$

S'agissant de Speroni, il semble que soit réglé le problème de cette «allusion », comme dit Alison Lovell. S'agissant maintenant de Platon, une relecture attentive du dialogue confirme cette absence, tant dans le discours de Diotime elle-même que dans celui des commentateurs internes à la fiction, à commencer par Socrate ${ }^{25}$. Une absence qui n'a rien d'étonnant si l'on songe que le cœur du sujet, et de l'enseignement ${ }^{26}$ que Diotime délivre à Socrate, c'est la juste représentation de l'amour, qui elle-même prélude à la juste représentation de la Beauté (l'ascension spirituelle depuis l'amour d'un beau garçon en particulier jusqu'à la contemplation du Beau en soi, détaché de toute particularité matérielle) : aux yeux d'un platonicien conséquent, la haine serait tout au plus une passion qui révèle notre faiblesse et notre petitesse en nous faisant les jouets d'une illusion. Socrate, dans le Phédon ou l'Apologie, avait tout lieu de haïr ses juges et accusateurs, mais, tout en se défendant hardiment, il donne le sentiment de les plaindre ${ }^{27}$. S'agissant enfin d'un dernier maillon capital dans la tradition platonicienne, maillon qui s'intercale entre le dialogue original et celui de Speroni, à savoir bien sûr le fameux Commentaire sur le banquet de Platon de Marsile Ficin (autrement appelé De Amore, et composé en 1469), auquel tout le monde songeait sans doute, force est de constater que la récolte est aussi maigre, malgré tout ce qu'ont pu ajouter à la strate platonicienne authentique les considérations médicales et théologiques du Moyen Âge chrétien. Dans le chapitre crucial, pas un seul instant la Diotime ficinienne ne mentionne ou ne présuppose la nécessité d'un sentiment de haine, fût-il dirigé

\footnotetext{
${ }^{24}$ Alison Lovell, «La Delie de Maurice Scève et le fond poétique italien du Commentaire sur le Banquet de Platon [De Amore] de Marsile Ficin », revue en ligne Cornucopia, Le Verger - III - Délie - décembre 2012, p. 11.

${ }^{25}$ Voir Le Banquet, texte établi et traduit par Paul Vicaire, 2e tirage revu et corrigé, 1992, 201d-212a (p. 51-71).

${ }^{26}$ Enseignement qu'il faut prendre au sens strict du terme: Socrate souligne l'aplomb magistral de l'Étrangère de Mantinée, son « ton le plus doctoral » (« $\check{\sigma \pi \varepsilon \rho \rho ~ o i ~ \tau \varepsilon ́ d \varepsilon o l ~ \sigma o \varphi ı \sigma \tau \alpha i ~ », ~ 208 c), ~ e t ~ s e ~ d i t ~ « ~ e n ~ a d m i r a t i o n ~ d e v a n t ~ s o n ~ s a v o i r ~ » . ~}$

${ }^{27} \mathrm{Il}$ s'amuse même à les provoquer dans l'Apologie, en déclarant estimer qu'il mériterait d'être entretenu à vie au Prytanée.
} 
contre les illusions $^{28}$. Il s'agit simplement d'insister sur l'approche bénéfique de la Beauté divine.

Cela ne signifie pas que Ficin élimine totalement le sentiment de haine de l'expérience amoureuse, mais c'est en tant que donnée funeste et limitée à l'amour charnel qu'il en reconnaît l'existence, et bien entendu cette haine ne fait pas l'objet d'un enseignement au sens strict du terme: Michael J. Giordano affirme d'ailleurs dans son dernier ouvrage que le précepte selon lequel la haine est indissociable de l'amour est néoplatonicien, se trouve chez Ficin et Speroni, mais, précise-t-il avec honnêteté, pas dans le discours de Diotime dans le Banquet de Platon ${ }^{29}$. Mis à part le terme de «précepte », déplacé ici ou légèrement impropre, il voit juste, car le Commentarium dit :

De plus l'amour bestial et l'amour humain ne peuvent jamais exister sans indignation. Qui ne s'indignerait pas contre celui qui lui a ravi son âme? Autant la liberté est agréable par-dessus tout, autant la servitude est accablante. C'est pourquoi tu détestes et tu aimes en même temps ceux qui sont beaux; tu les hais en tant que voleurs et homicides et tu es contraint de les admirer et de les aimer comme des miroirs reflétant un éclat céleste ${ }^{30}$.

Un tel propos réactive dans une perspective clairement spiritualiste le très ancien Odi et amo catulléen $^{31}$, en substituant à l'absence d'explication - on se souvient que le locuteur romain ne s'explique pas cette contradiction : "Quare id faciam, fortasse requiris. / Nescio» - une logique relationnelle de l'Amant avec sa propre liberté et non pas seulement avec l'être aimé. Et de fait, n'était la mention explicite de «Dyotime », la tonalité du dizain scévien, dans sa chute, rappelle plutôt la rigueur et la violence paradoxale de Catulle, - un Catulle qui, pour certains spécialistes, ne serait cependant pas forcément éloigné d'une sensibilité platonicienne en dépit de son goût de l'outrance et de la grossièreté, car il est à la recherche d'un paradis perdu, d'une anamnèse salvatrice contre la laideur du monde ${ }^{32}$.

En tout cas cet examen des intertextes ne fait que renforcer l'apparente contradiction, et la valeur provocatrice du concetto, car un dilemme finit par s'imposer : ou bien l'Amant scévien éprouve réellement de la haine aussi bien que de l'amour, mais alors une Diotime digne de ce nom ne saurait la lui enseigner; ou bien cette Diotime lui enseigne bien une haine, et alors

\footnotetext{
${ }^{28}$ Voir Marsile Ficin, Commentaire sur le Banquet de Platon, éd. Raymond Marcel, 1978, chap. 18, p. 235-239.

29 «The notion that hate is a natural concomitant of love is another Neoplatonic precept that one will see in Ficino and in Speroni (but not in Diotima's speech in Plato's Symposium)» (Michael J. Giordano, The Art of Meditation and the French Renaissance Love Lyric, op. cit., p. 399).

${ }^{30}$ Commentaire sur le Banquet de Platon, éd. cit., p. 222. Texte original : « Accedit ad hec quod et ferinus et humanus amor sine indignatione esse numquam potest. Quis illi non indignetur, qui ipsi abstulerit animum? ut enim pre ceteris grata libertas ita servitus onerosa. Itaque formosos odis simul et / amas odis tamquam fures et homicidas, tamquam specula celesti fulgore micantia mirari cogeris et amare. $»\left(\mathrm{VI}, 10,84 \mathrm{r}^{\circ}-84 \mathrm{v}^{\circ}\right)$.

${ }^{31}$ Catulli Veronensis Liber, 85.

${ }^{32}$ Voir par exemple la préface de Jean-Pierre Néraudau, dans Catulle, Poésies, 1998, p. XXIV-XXV.
}

https://journals.uvic.ca/index.php/sator/index 
c'est une haine qui n'en est pas vraiment une, qui dépasse le sens obvie et qu'il faut prendre figuralement ; pour lever le doute, il faut maintenant enquêter au sein du recueil et y observer les autres emplois.

Celui-ci présente six autres occurrences du vocable de « hayne », ou « haïr » : tout d'abord deux occurrences peu pertinentes, car relativement lexicalisées : au D 15, l'Amant se réjouit que le « vil Siecle avare » soit passé sous l’influence quasi thérapeutique de Délie,

Et plus ne hayt l'honneste estrangement, Commençant jà a cherir la vertu.

La « haine » en question ici est une détestation ordinaire et lâche de l'honnêteté par des contemporains bassement matérialistes. Le terme est manifestement moins fort que dans le D 439 : affaire de goût, d'option dans le comportement, de sensibilité. Seconde occurrence peu significative, au D 391 l'incendie de Lyon $^{33}$ est imputé à Vénus, en rétorsion contre l'ingratitude des habitants :

Les Dieux hayantz ingratitude vile,

Nous font sentir double vengeance amere.

Ce contexte de violence punitive rend le verbe « haïr » synonyme de « ne pas supporter », « avoir une (sainte) horreur de», et se fait d'autant moins remarquer que son complément redondant (« ingratitude vile ») le fait aller de soi.

Mais quatre autres occurrences sont beaucoup plus utiles à la clarification contextuelle du D 439. Le D 60, en particulier, met le verbe en système :

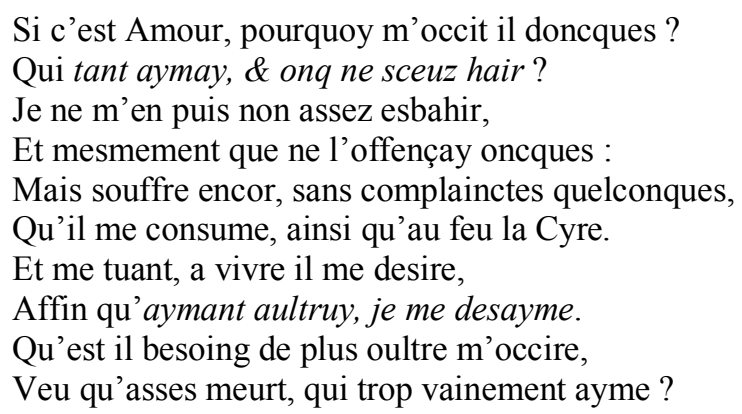

Un grand nombre de différences avec les emplois précédents sont à remarquer : ici, en effet, le verbe

- est à l'infinitif comme au D 439 (d'où sa quasi-nominalisation) ;

\footnotetext{
${ }^{33}$ En 65 ap. J.-C. Voir Sénèque, Lettres à Lucilius, XCI.
}

https://journals.uvic.ca/index.php/sator/index 
- est en régime absolu, comme au D 439 ;

- est régi par le « Je » de l'Amant, et non par une tierce personne (le « vil siècle avare », « les Dieux ») ;

- se trouve en système avec le verbe « aimer » comme au D 439;

- est complément d'objet direct de « savoir », ce qui présuppose l'apprentissage ou du moins la connaissance, la conscience d'une pratique, d'un comportement. Certes, ce «savoir » est atténué par l'auxiliarité, mais la sémantèse première n'est pas absolument éradiquée (le test de substitution avec « vouloir » ou « pouvoir » est probant car il donne une autre image : * onq ne vouluz hayr », * « onq ne pus hayr » disent tout autre chose).

- est très voisin d'un autre verbe présent au D 439, « esbahir » (v. 3), pris de surcroît comme là dans une formule impliquant le « Je » de l'Amant-poète.

Dans une logique très proche, le D 315 s'ouvre sur un vers mystérieux mais où le système « aimer / haïr » est reconduit :
Je m'ayme tout au desdaing de la hayne,
Ou toutesfois je ne l'ose irriter,
Si doulcement elle est de courroux plaine,
Que contre soy se prent a despiter :
Dont tout plaisir je me sens conciter,
Et n'est possible en fin que je m'en taise.
Parquoy couvrant en mon cœur ce grand aise,
Qui ne me peult detenir en ma peau,
Je vois a elle, \& m'accuse, \& l'apaise,
Lors l'air troublé soudain retourne en beau.

Ian Mc Farlane pense que la «hayne » en question est celle des « envieux » du dizain précédent, ce qui est ingénieux, car les micro-séquences existent, mais peu probable à cause de la suite immédiate, qui en donne une raison suffisante. L'explication du vers tient à la valeur probablement circonstancielle de l'adverbe «tout», synonyme ici de «quand je suis

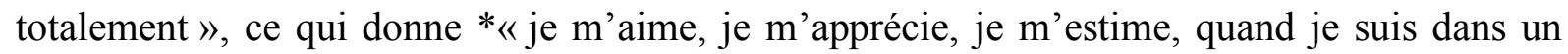
parfait mépris («tout au dédain ») vis-à-vis de la haine que Délie pourrait ressentir envers moi, mais à laquelle cependant je me garde bien de l'inciter ${ }^{34}$. La « hayne » en question est donc ici celle que pourrait éprouver Délie à son égard, mais on ne voit pas en quoi elle pourrait faire l'objet d'un quelconque magistère.

\footnotetext{
${ }^{34}$ Même analyse chez G. Defaux dans son édition (t. II, p. 355)

https://journals.uvic.ca/index.php/sator/index
} 
En revanche, le D 401 présente une conséquence autodestructrice de la contemplation que fait 1'Amant des qualités remarquables (« conditions $\left.^{35} »\right)$ de Délie plus suggestive : ses esprits à lui sont tellement bouleversés, transformés au vu de ces qualités immenses...

... [Qu'] en me hayant de toute hayne extreme, Comme me hayt sa gracieuseté, Je me suis fait ennemy de moymesme, Pour tout complaire a son impiété.

(Où l'on voit au passage que le « tout » a ici aussi la valeur adverbiale de « entièrement »). Ce processus «psychérologique » donne tout de même une piste très intéressante, car c'est le propre d'un « ennemi » que de « haïr », et parce que, pour une fois, est clairement mentionnée la haine que Délie peut éprouver pour l'Amant. Il n'y a évidemment toujours pas trace d'une formation délibérée à la haine dispensée par la première au second, hormis sans doute, et il ne faut pas y voir un détail sans importance, la présence de ce «comme » qui suggère une imitation au moins autant qu'un simple parallèle. Plus exactement, ce «comme» permet, autorise, laisse lire, une application mimétique. La haine que la "gracieuseté » de Délie voue à l'Amant est prise à son compte et imitée par celui-ci, qui, pour tenter de la convertir en faveur, se déteste lui-même. Ce statut d'heautontimoroumenos rejoint les nombreux topoï plus ou moins gnomiques sur la nécessaire mort à soi-même, sur la dialectique du regard de l'Autre comme plus fidèle miroir, de la nuit d'ici qui est le jour aux antipodes, sur le remède pire que le mal, etc. ${ }^{36}$. Mais reste un écueil identificatoire : comment Diotime pourrait-elle jouer ici ce rôle (même sans être explicitement nommée, disons une « Délie-Diotime »), alors que sa grâce voue de la haine à l'Amant, et que, surtout, c'est non pas à sa pureté, mais à son « impiété » qu'il s'agit de plaire ? Comment la même « impie » pourrait-elle être ensuite qualifiée de « tressaincte, \& sage »?

La dernière occurrence vraiment pertinente est celle du D 43 :

\footnotetext{
Moins je la voy, certes plus je la hays. Plus je la hays, \& moins elle me fasche. Plus je l'estime, \& moins compte j'en fais : Plus je la fuys, plus veulx, qu'elle me sache. En un moment deux divers traictz me lasche Amour, \& hayne, ennuy avec plaisir. Forte est l'amour, qui lors me vient saisir, Quand hayne vient \& vengeance me crie : Ainsi me faict hayr mon vain desir Celle, pour qui mon cœur tousjours me prie.
}

\footnotetext{
${ }^{35}$ Voir le passage de Speroni cité supra, p. 5.

${ }^{36}$ Voir par exemple les D 49, D 257, D 446.
}

https://journals.uvic.ca/index.php/sator/index 
Cette suite de paradoxes et d'apories en balance, compliquée de chiasmes, et qui recycle la vieille opposition de la flèche d'or et de la flèche de plomb de Cupidon ${ }^{37}$, s'achève sur un quatrain dont trop peu de commentateurs ont noté l'ambiguïté syntaxique due à la possibilité de l'inversion sujet / c.o.d. : le lecteur peut hésiter, pour le sens des deux derniers vers, entre comprendre «mon vain désir me fait haïr celle qui... », donc haïr Délie par périphrase, et comprendre «Celle pour qui...[donc Délie] me fait haïr mon vain désir ». La première solution serait plus cohérente par rapport à tout ce qui précède, et la haine serait directement intelligible : l'Amant passe par tant d'états contraires et paradoxaux que son vain désir lui fait haïr celle pour qui son cœur le mobilise en permanence ; l'amour passionné, parce qu'il est passionnel et donc douloureux, engendre la haine de l'être aimé, qui est cause de tant de souffrances (et serait donc relancé le cercle vicieux de la haine dont il était question tout à l'heure chez Ficin : l'Amant en veut au voleur de son cœur). Mais au niveau du cotexte plus ramassé, c'est la seconde qui l'emporte, comme je l'ai déjà expliqué ailleurs ${ }^{38}$, et le sens est au moins aussi satisfaisant, quoique radicalement différent : la vanité du désir de l'Amant est ce qui explique toute cette série de paradoxes et de mécanismes aberrants ; partant, l'action concrète de Délie sur l'Amant consiste à lui faire identifier le responsable, ce «vain désir », justement, qui empêche un véritable « amour», dont elle est l'ambassadrice. Là, en revanche, il y a une place pour une figure de Diotime, la seule distinction persistante avec le personnage tutélaire étant que la femme de Mantinée, elle, n'est pas l'objet d'un amour passionnel.

Est-il possible dès lors, compte tenu de tous ces éléments, d'esquisser une synthèse, et de dégager sans la moindre hésitation le véritable sens de cette spectaculaire assimilation de Délie à une Diotima rediuiua qui enseignerait aussi bien la haine que l'amour? Quelle Diotime, quelle haine et quel amour?

La réponse ne sera possible qu'à condition de tenir compte de deux derniers éléments de contextualisation: d'une part, le fait que l'assimilation de Délie à Diotime est la dernière d'une longue série qui comprend notamment Pandora (D 2) et Hécate (D 22), mais surtout d'une série de personnages féminins dont le nom débute étrangement par la lettre D : « Delie » (D 22, D 335 et passim) évidemment, «Delia » (D 131), «Daphnes » (D 102), « Diane » (D 176), « Dictymne» (D 353), et accessoirement « Dame » (D 63) et «Déesse» (D 105, 322). Variantes mythologiques de l'objet de désir, dont certains ont pu dire - comme

\footnotetext{
${ }^{37}$ Voir Ovide, Métamorphoses, I, 466-471.

${ }^{38}$ Voir Nathalie Dauvois, Michèle Clément, Xavier Bonnier, Maurice Scève. Délie, 2012, p. 208-209.
}

https://journals.uvic.ca/index.php/sator/index 
Jacqueline Risset - qu'elles étaient tentative d'épuisement du signifiant « désir » lui-même ${ }^{39}$. « Dyotime » étant la toute dernière persona de Délie, il est permis de supposer que c'est au moins partiellement sur cette image que Scève désire que reste le lecteur, si le cheminement du recueil a un sens; et bien entendu terminer sur Diotime c'est terminer sur une certaine révélation finale, sur une sagesse difficilement gagnée. Mais d'autre part, et c'est là que le bât blesse, toutes les autres personce sont des proies virtuelles de l'Amant, dont seule la beauté vierge et farouche attire, et qui peuvent rester muettes. Tel n'est absolument pas le cas de Diotime, qui n'a rien d'une vierge effarouchée, dont seul le langage a un sens, et qui pourrait donc au moins avoir droit au discours direct, comme il arrive souvent que Délie s'exprime à la première personne dans des saynètes de fantaisie ${ }^{40}$ : là où Diane se contente de punir les curieux, là où Daphné et Dictymne s'enfuient à toutes jambes, Diotime est censée produire du discours, donner verbalement un cours accéléré sur l'amour qu'on aurait plaisir à entendre, surtout au terme d'un parcours aussi erratique; or, à tous les sens du terme et pour jouer sur les mots, la Diotime scévienne est aussi bien «sans leçon» que «sans le son », c'est un mentor de circonstance sagement tenu en bride par son prétendu disciple, et dont on n'entend pas un traître mot.

Comme souvent chez Scève, le texte reprend d'une main ce qu'il donne de l'autre, les énoncés s'auto-contredisent ou se neutralisent, et les réponses sont plus ténébreuses que les questions. Mais ce n'est pas qu'un jeu de roublardise virtuose dépourvu d'enjeu : tout est configuré, dans ce dizain, pour que le mentorat de Diotime soit piégé, voire totalement irreprésentable. Parce que le quatrain explique le sizain, et que c'est manifestement l'inaudible parole de Délie-Diotime qui cause le désordre chez l'Amant au lieu d'y porter remède; parce que persiste une haine qui dans le meilleur des cas est dirigée contre les faiblesses libidinales de l'Amant, et compromet donc la contemplation puisqu'elle engage une lutte ; parce que les dizains suivants font alterner le meilleur idéalisme (D 440, 445) et la rage indignée la plus virulente (D 441, 447), quand ce n'est pas le doute pur et simple (D 448), ce qui interdit l'ataraxie; parce que le tout dernier dizain du recueil n'affirme pas la suprématie de la «vertu » sur l'« ardeur », ce qui serait très platonicien, mais leur subversive équation dans le cadre de l'œuvre passée à la postérité des siècles à venir ; parce que Diotime est désirée alors qu'elle est inaccessible, « tressaincte, \& sage », et ne peut donc délivrer d'autre leçon que muette, lointaine et inassimilable, très au-dessus des forces "suffoquées » de

\footnotetext{
${ }^{39}$ Voir Jacqueline Risset, L'Anagramme du Désir. Sur la Délie de Maurice Scève, 1995, p. 125-131.

${ }^{40}$ Voir par exemple le D 109 (Délie s'emparant de l'épée de Mars) ou le D 332 (la chute du dé à coudre) : sa parole est toujours vive, dense et décidée.
} 
l'Amant. La conséquence de cette conséquence, c'est qu'il n'y a nulle allégeance et nulle sujétion: l'aliénation qui rive l'Amant à son reliquat passionnel est le masque d'une formidable liberté qui, elle, est la seule vertu possible. La nécessaire culture de l'entre-deux, de l'équilibre instable et de l'inconfort, fait briller au plus haut une Diotime qui est elle-même manipulée par le poète non pas en tant qu'Amant, mais en tant que poète. Comme s'il ne fallait à celui-ci d'autre mentor que lui-même.

Xavier Bonnier Université de Rouen Normandie CÉRÉdI (EA 3229)

\section{BIBLIOGRAPHIE}

ALDUY, Cécile, « Les « erreurs » de la versification dans Délie de Maurice Scève », Nouvelle Revue du Seizième siècle, 16/2, 1998, p. 249-266.

DAUVOIS, Nathalie, Michèle CLÉMENT, Xavir BONNIER, Maurice Scève. Délie, Neuilly-sur-Seine, Atlande, 2012.

DEFAUX, Gérard, Delie object de plus haulte vertu, Genève, Droz, 2004.

DUPRIEZ, Bernard, Gradus - Les procédés littéraires (Dictionnaire), Paris, UGE, (10/18), 1980.

DUVAL, Edwin M., « From the Chanson parisienne to Scève's French canzoniere : Lyric Form and Logical Structure of the Dizain », dans Jerry C. NASH (éd.), A Scève Celebration : Délie 1544-1994, Saratoga, Anma Libri, 1994, p. 71-85.

FENOALTEA, Doranne, "The Final Dizains of Sc ève 's "Délie" and the "Dialogo d'Amore" of Sperone Speroni », Studi Francesi, 59, 1976, p. 201-225.

FICIN, Marsile, Commentaire sur le Banquet de Platon, Paris, Les Belles Lettres (Les classiques de l'humanisme), 1978. [R. Marcel (éd)]

GIORDANO, Michael J., The Art of Meditation and the French Renaissance Love Lyric. The Poetics of Introspection in Maurice Scève's Délie, Object de plus haulte vertu (1544), Toronto, University of Toronto Press, 2010. 
LOVELL, Alison, « La Delie de Maurice Scève et le fond poétique italien du Commentaire sur le Banquet de Platon [De Amore] de Marsile Ficin », revue en ligne Cornucopia, Le Verger - III - Délie - décembre 2012

http://cornucopia16.com/wp-content/uploads/2014/07/VBQIII-LOVELL.pdf

McFarlane, IAN, The « Délie » of Maurice Scève, Cambridge University Press, 1966.

CATULlE, Poésies, Paris, Les Belles Lettres (Classiques en poche), 1998. [J.-P. Néraudau (éd.)]

PÉTRARQUE, Canzoniere / Le Chansonnier, Paris, Classiques Garnier / Bordas, 1988. [P. Blanc (éd.)]

PLATON, Le Banquet, Paris, C.U.F., Les Belles Lettres, 1992. [P. Vicaire (éd.)

RISSET, Jacqueline, L 'Anagramme du Désir. Sur la Délie de Maurice Scève, Paris, Fourbis, 1995.

SCÈVE Maurice, Délie object de plus haulte vertu, Paris, STFM, 2001. [E. Parturier (éd.)]

SPERONI, Sperone, Dialogo d'amore, trad. Claude Gruget, Dialogue traittant d'amour \& jalousie, Poitiers, La Licorne (Textes rares), 1998. [P. Martin (intr.)] 